\title{
The brain tissue response to implanted silicon microelectrode arrays is increased when the device is tethered to the skull
}

\author{
Roy Biran, ${ }^{1}$ Dave C. Martin, ${ }^{2}$ Patrick A. Tresco ${ }^{1}$ \\ ${ }^{1}$ The Keck Center for Tissue Engineering, Department of Bioengineering, College of Engineering, \\ University of Utah, Salt Lake City, Utah 84112 \\ ${ }^{2}$ Macromolecular Science and Engineering Center, Department of Materials Science and Engineering, \\ University of Michigan, Ann Arbor, Michigan 48109
}

Received 14 November 2005; revised 14 September 2006; accepted 26 September 2006

Published online 31 January 2007 in Wiley InterScience (www.interscience.wiley.com). DOI: 10.1002/jbm.a.31138

\begin{abstract}
The influence of tethering silicon microelectrode arrays on the cortical brain tissue reaction was compared with that of untethered implants placed in the same location by identical means using immunoflourescent methods and cell type specific markers over indwelling periods of 1-4 weeks. Compared with untethered, freely floating implants, tethered microelectrodes elicited significantly greater reactivity to antibodies against ED1 and GFAP over time. Regardless of implantation method or indwelling time, retrieved microelectrodes contained a layer of attached macrophages identified by positive immunoreactivity against ED1. In the tethered condition and in cases where the tissue surrounding untethered implants had the highest levels of ED1+ and GFAP+ immunoreac-
\end{abstract}

tivity, the neuronal markers for neurofilament 160 and NeuN were reduced. Although the precise mechanisms are unclear, the present study indicates that simply tethering silicon microelectrode arrays to the skull increases the cortical brain tissue response in the recording zone immediately surrounding the microelectrode array, which signals the importance of identifying this important variable when evaluating the tissue response of different device designs, and suggests that untethered or wireless devices may elicit less of a foreign body response. (C) 2007 Wiley Periodicals, Inc. J Biomed Mater Res 82A: 169-178, 2007

Key words: implant; silicon electrode; tissue response; foreign body response; neuroprosthetic

\section{INTRODUCTION}

Implanted electrodes are routinely employed in various research applications for recording and stimulating nervous tissue. High density, multisite electrode arrays represent a promising area of biomedical device development. ${ }^{1}$ Already, multisite stimulating electrodes are being used for the treatment of deafness ${ }^{2}$ as well as to alleviate symptoms of Parkinson's disease. ${ }^{3,4}$ In addition, advances in our understanding of the functional architecture of the central nervous system (CNS) have been facilitated by the availability of multisite recording electrodes. ${ }^{5}$

The emergence of micromachining technology has yielded increasingly smaller and more sophisticated

Correspondence to: P.A. Tresco; e-mail: patrick.tresco@ utah.edu

Contract grant sponsor: NIH/NINDS; contract grant numbers: N01-NS-1-2338, R01 NS046770 electrodes capable of recording from greater volumes of neural tissue with improved spatial resolution. ${ }^{6,7}$ Despite substantial technological advances in hardware and design, many devices perform inconsistently in chronic applications. ${ }^{8}$

Available evidence suggests that the brain tissue reaction is responsible for the degradation of recording performance. Studies conducted over three decades have demonstrated that encapsulation tissue eventually surrounds implanted electrodes, as occurs with other types of materials implanted in the nervous system, which contains a variety of cell types and their secreted matrix including meningeal fibroblasts, macrophages, and reactive astrocytes that appear to vary both in spatial distribution and in abundance over time. ${ }^{9-16}$ Current theories regarding the mechanism of recording instability and degradation suggest that encapsulation of the electrode by reactive astrocytes acts to electrically insulate the electrode from nearby neurons, ${ }^{8,9,15,16}$ possibly hindering diffusion ${ }^{17}$ and/or increasing the distance between the electrode and adjacent neurons. ${ }^{8}$ 
While much attention has been placed on characterizing the astrocytotic response to microelectrode implantation, less attention has focused on the fate of nearby neurons. A recent report observed that neuronal loss accompanies the foreign body response to chronically implanted silicon microelectrode arrays, which appears to be separate from the cell loss that is caused by the initial implantation trauma. ${ }^{18}$ The investigators observed persistent ED1 immunoreactivity in the tissue surrounding silicon microelectrode arrays implanted in rat brain tissue, which was accompanied by a reduction in nerve fiber density and loss of nerve cell bodies. Persistent upregulation of ED1 and neuronal cell loss was not observed in microelectrode stab wound used as controls, suggesting that the foreign body response plays an important role in the loss of neurons. In addition, explanted electrodes were covered with ED1/ MAC1 positive cells that released both pro-inflammatory and neurotoxic molecules in vitro, which prompted the authors to speculate that inflammation-associated neurotoxicity may be another factor contributing to the loss of recording performance.

While the literature reflects a concerted effort to identify and characterize the changing characteristics of the foreign body response around various types of implanted electrodes, most studies have focused on fully functional implants or on passive, nonfunctional implants using a variety of anchoring methods. At present, it is unclear whether the manner in which an electrode is anchored has an impact on the foreign body response. To address this issue, the current study was designed to study the influence of electrode tethering mechanism on the adjacent brain tissue reaction over time by comparing different models of anchoring the same planar silicon microelectrode array in rat cortex, a target tissue for neural prosthetic development. ${ }^{19}$ Our results indicate that the method of anchoring an electrode in brain tissue (tethering) significantly affects the brain tissue response and suggests that freely floating (wireless) electrodes may elicit less brain tissue reactivity than present designs that require the passage of electrically conductive materials through an opening in the skull to interact with electrical devices outside the body.

\section{MATERIALS AND METHODS}

\section{Microelectrodes}

Silicon microelectrodes fabricated for chronic implantation were supplied by the Center for Neural Communication Technology (CNCT) at the University of Michigan (http://www.engin.umich.edu/facility/cnct/). All electrodes contained a single shank with the following dimensions: length, $5 \mathrm{~mm}$; width, $200 \mu \mathrm{m}$ at the base tapering to
$33 \mu \mathrm{m}$ at the tip; thickness, $15 \mu \mathrm{m}$ along the shank and $2 \mu \mathrm{m}$ at the tip. All electrodes were cleaned by immersion in $70 \%$ ethanol, followed by rinsing in sterile, deionized water, and sterilized prior to implantation by ethylene oxide.

\section{Animal surgery}

All procedures involving animals were conducted using sterile technique in accordance with protocols approved by the University of Utah Institutional Animal Care and Use Committee and NIH guidelines for the care and use of laboratory animals. Adult male Fischer 344 rats $(225-250 \mathrm{~g})$ were anesthetized with a cocktail of ketamine $(65 \mathrm{mg} / \mathrm{kg})$, xylazine $(7.5 \mathrm{mg} / \mathrm{kg})$, and acepromazine $(0.5 \mathrm{mg} / \mathrm{kg})$. Upon reaching full depth of anesthesia, the eyes were covered with ophthalmic ointment and the head was shaved. The scalp was disinfected by treatment with isopropanol, followed by liberal treatment with butadiene. Animals were transferred to a stereotaxic frame (Stoelting, Wood Dale, IL) set under a stereomicroscope. A midline incision extending the length of the skull was made. For all implant types, a 3-mm-diameter burr hole was created through the skull with a trephine drill bit held and lowered under stereotactic control and driven pneumatically. The center of the hole was positioned at coordinates $+0.2 \mathrm{~mm}$ forward of bregma and $3 \mathrm{~mm}$ lateral to bregma. While drilling, sterile PBS was applied to the bit with a $10-\mathrm{cm}^{3}$ syringe through a 25G needle to cool the drill site and reduce heat transfer to the underlying cortical tissue. The bone plug was removed with fine forceps under stereomagnification. The hole was rinsed liberally with sterile PBS to remove drill debris and the dura was carefully opened with a 21G needle. The various implant methods employed are described later and shown in Figure 1.

\section{Untethered microelectrodes}

Untethered microelectrodes were held with a pair of fine forceps and lowered into the brain by hand to a depth of $2 \mathrm{~mm}$. The top of the microelectrode was severed with scissors so that the entire electrode shaft was implanted in brain tissue leaving the top of the electrode flush with the surface of the cortex. The bone plug was replaced, and the scalp sutured with 5/0 silk sutures (Fig. 1).

\section{Conventionally tethered microelectrodes}

Another group of microelectrodes was implanted using a conventional method as employed in chronic recording experiments, two bone screws were attached to the skull $\sim 4-5 \mathrm{~mm}$ on either side of the sagital suture in the temporal bone. A single microelectrode with attached connector was carefully lowered into the brain at coordinates using a modified stereotactic micromanipulator. The electrode was secured with silicone elastomer (Kwik-cast; World Precision Instruments, Sarasota, FL). Acrylic dental adhesive (Dura Lay; Dental Mfg., Worth, IL) was applied as a slurry around the bone screws to cover the skull, and the connector was lowered into position on the acrylic cement. Addi- 


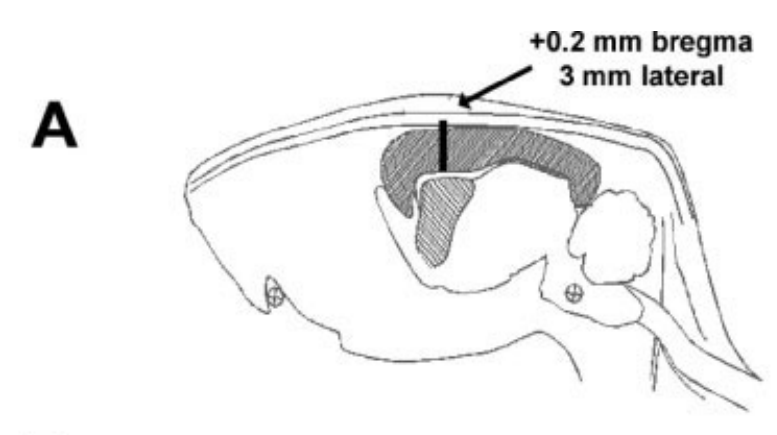

B
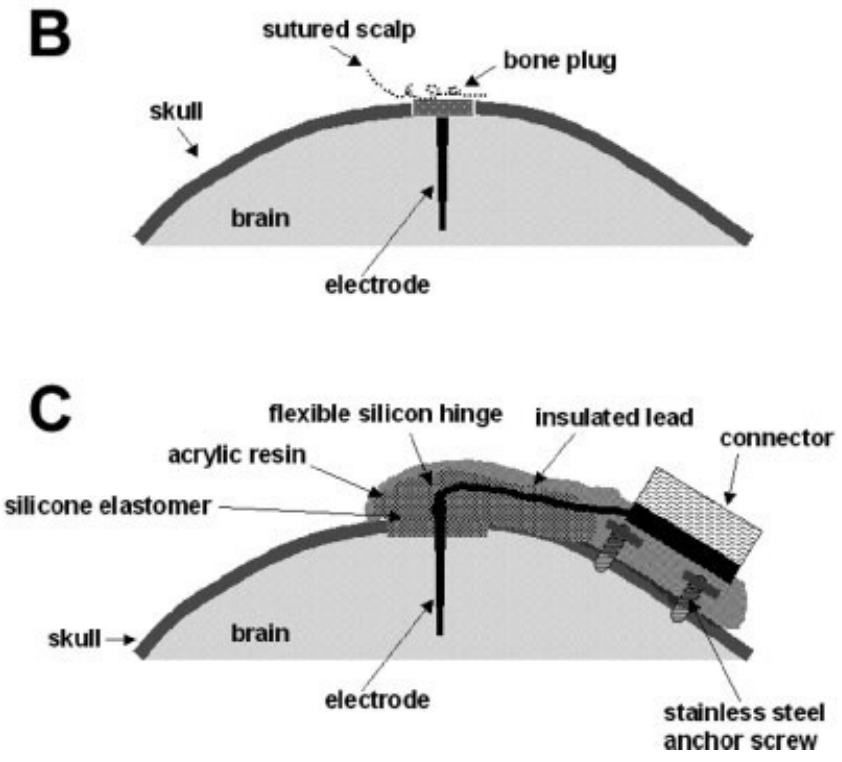

Figure 1. Diagram of different implantations schemes. (A) Microelectrodes were lowered into the cerebral cortex at the indicated stereotaxic coordinates. (B,C) Diagrams indicating the implantation method and materials used for (B) untethered silicon microelectrode arrays and (C) conventionally tethered silicon microelectrode arrays.

tional acrylic was applied with a $1-\mathrm{cm}^{3}$ syringe to surround and secure the connector to the screws and also to capture the connection to the microelectrode array (Fig. 1). The scalp was left to heal around the fixture.

\section{Euthanasia and tissue processing}

At various time points after implantation, animals were euthanized with a mixture of ketamine $(70 \mathrm{mg} / \mathrm{kg})$ and xylazine (30 mg/kg). Animals were perfused transcardially at a flow rate of $50 \mathrm{~mL} / \mathrm{min}$ with a minimum of $125 \mathrm{~mL}$ of ice-cold PBS, followed by $125 \mathrm{~mL}$ of fresh ice-cold $4 \%$ (w/v) paraformaldehyde. Following perfusion, each brain was carefully removed from the skull. Brains were postfixed overnight in $4 \%$ paraformaldehyde in PBS. Microelectrodes were carefully retrieved with sharp microdissection forceps and processed for immunostaining.

\section{Histology and immunostaining}

Brains were cut in horizontal sections of $40 \mu \mathrm{m}$ thickness with a Vibratome (Vibratome, St. Louis, MO). A list of antibodies used, their source, and dilution is detailed in Table I. All antibodies were diluted in blocking solution consisting of $4 \%(\mathrm{v} / \mathrm{v})$ normal goat serum, $0.3 \%(\mathrm{v} / \mathrm{v})$ Triton-X-100, and $0.1 \%(\mathrm{w} / \mathrm{v})$ sodium azide. Approximately 6-8 tissue sections at the level of the cerebral cortex per animal were immunostained for each marker. The sections for each staining set spanned $\sim 1.8 \mathrm{~mm}$ of the cerebral cortex, with each section separated from the next by about $300 \mu \mathrm{m}$ distance. Sections were incubated for $1 \mathrm{~h}$ with blocking solution at room temperature. Primary antisera were applied overnight at $4^{\circ} \mathrm{C}$. After three rinses in PBS (15 min/rinse), appropriately matched secondary antibodies were applied for $1 \mathrm{~h}$ at room temperature. ED1 and MAC-1 (OX-42) stains were amplified by applying a biotinylated secondary antibody, followed by incubation with fluorescently labeled streptavidin for $1 \mathrm{~h}$. Alexa-488 and Alexa-594 labeled secondary antibodies and streptavidin were purchased from Molecular Probes (Eugene, OR). Biotinylated secondary antibodies were purchased from Southern Biotech (Birmingham, AL). Secondary antibodies were diluted in blocking solution as follows: Alexa-488/ 594 secondaries, $10 \mu \mathrm{g} / \mathrm{mL}$; biotinylated secondaries, $5 \mu \mathrm{g} / \mathrm{mL}$; Alexa-488-streptavidin, $2.5 \mu \mathrm{g} / \mathrm{mL}$. After washing with PBS, sections were counterstained with $10 \mu M$ DAPI (Molecular Probes) mounted onto microscope slides using Fluoromount-G (Southern Biotech) and covered with a coverglass. Retrieved electrodes were treated in a similar manner.

\section{Imaging and quantification}

For quantification of the spatial distribution of antigens, digital images were collected using a Coolsnap color CCD

TABLE I

Summary of Antibodies

\begin{tabular}{|c|c|c|c|c|c|}
\hline Antibody & Antigen & Cell Type(s) & Isotype & $\begin{array}{c}\text { Concentration } \\
(\mu \mathrm{g} / \mathrm{mL})\end{array}$ & Vendor \\
\hline CD68 (ED1) & Lysosomal glycoprotein & Microglia, macrophages & Mouse IgG1 & 1.0 & Serotec \\
\hline CD11b/c (OX-42) & CR3 complement receptor & Microglia, macrophages & Mouse IgG2 $\alpha$ & 2.5 & Pharmingen \\
\hline GFAP & Glial fibrillary acidic protein & Astrocytes & Rabbit IgG & 4.0 & Dako \\
\hline Vimentin & $\begin{array}{l}\text { Intermediate filament, } \\
\text { vimentin }\end{array}$ & $\begin{array}{l}\text { Astrocytes, fibroblasts, } \\
\text { microglia }\end{array}$ & Mouse IgG1 & 6.0 & Sigma \\
\hline Neurofilament-160 & $\begin{array}{l}\text { Medium neurofilament } \\
\text { polypeptide }\end{array}$ & Neurons & Mouse IgG1 & 2.4 & Sigma \\
\hline NeuN & Neuronal nuclei & Neurons & Mouse IgG1 & 5.0 & Chemicon \\
\hline
\end{tabular}


TABLE II

Animal Numbers in Each Category

\begin{tabular}{ccc}
\hline Duration (weeks) & Untethered & Conventional Tether \\
\hline 1 & 9 & 6 \\
2 & 9 & 6 \\
4 & 8 & 6 \\
Total & 26 & 18 \\
\hline
\end{tabular}

(Roper Scientific, Trenton, NJ) attached to a Nikon E600 microscope using Image Pro 4.5 software (Media Cybernetics, Silver spring, MD), using the method as previously described in Ref. 18.

\section{Animal numbers and statistical analysis}

A total of 44 adult male Fischer 344 rats (225-250 g) were used as described in Table II. The reactive distance for GFAP was established arbitrarily as the average distance where the relative intensity of immunoreactivity was three times the background. To assess differences in immunoreactivity between conditions, comparisons of mean intensity values at discrete distances were compared by a two-tailed Students $t$ test with significance set at $p<0.05$.

\section{RESULTS}

All animals survived implantation with no complications.

\section{Comparison of conventionally tethered and untethered microelectrodes}

In order to assess the extent of inflammation between untethered and conventionally tethered microelectrodes, we examined the presence of macrophages using indirect immunofluorescence for ED1, a glycoprotein enriched in the lysosomal membranes of phagocytically active macrophages and microglia. ${ }^{20}$ Immunopositive cells, predominantly ameboid in morphology, were observed adjacent to the implantation site and observed throughout nearby tissue of both types of implants. ED1+ cells containing short processes were also observed hundreds of micrometers away from the implantation tract, as has been described by others, ${ }^{16}$ and often times were associated with vasculature, suggesting extravasation was ongoing throughout this period. In some specimens, ED1 immunoreactivity was observed within the implant cavity, which presumably resulted from cells being scraped off the surface of the electrode upon retrieval. Autofluorescent hemosiderin deposits were also observed within and immediately near some but not all implant cavities at the 7-day time point. The deposits appeared dark under light microscopy and contained no definable DAPI-stained nuclei under fluorescence. There was substantial var- iability in the amount of hemosiderin at implant sites of different animals with no apparent connection to the implant anchoring method, presumably resulting from variable vascular damage induced by implantation trauma.

At all time points, conventionally tethered microelectrode sites contained a significantly greater abundance and relative intensity of ED1 immunoreactivity compared with untethered, freely floating implants (Fig. 2). ED1 reactivity around the implantation site was readily notable at 1 and 2 weeks, often extending over an area as much as half a millimeter in diameter. The average distance around conventionally tethered electrodes was greater at each time point by approximately threefold at 1 week, and 10-fold greater at 2 and 4 weeks, respectively (Fig. 2, bottom panel). By 4 weeks, the reaction around untethered electrodes had subsided to only a thin band of ED1+ cells surrounding the implant (Fig. 2).

The extent of ED1 immunoreactivity around both implant types diminished over time (Fig. 2). By 4 weeks the average distance of ED1 immunoreactivity around conventionally tethered microelectrodes had dropped to approximately half of its initial value. In neither condition did ED1 immunoreactivity return to a normal unreactive state, that is, one with no observable ED1+ immunoreactivity. Thus, ED1 immunoreactivity was greater in the conventionally tethered group compared with the same biomaterial implanted without a tethering mechanism. While the extent of macrophage activation decreased over time and occupied a smaller zone surrounding the microelectrodes, ED1+ immunoreactivity never disappeared around either type of microelectrode.

\section{Conventionally tethered microelectrodes produce greater GFAP immunoreactivity than untetherd, freely floating microelectrode arrays}

Analysis of the GFAP immunoreactivity over time showed that hypertrophied intensely labeled astrocytes, like those observed around cortical stab wounds, ${ }^{21}$ surrounded both conventionally tethered and untethered implants (Fig. 3), often forming a dense band at a distance removed from the implant interface. At every time point, the extent of GFAP immunoreactivity was significantly greater around conventionally tethered electrodes compared to the untethered cohort. For both types of implants, the reactive band of astrocytes was never observed directly opposing the implant interface, but resided outside the ED1+ immunolabeled zone. After week 2 , there appeared to be little change in the extent of GFAP labeling observed around untethered microelectrodes that rarely formed a dense tight capsule. However, in the tethered cohort while the extent of the response significantly declined it often collapsed 
untethered
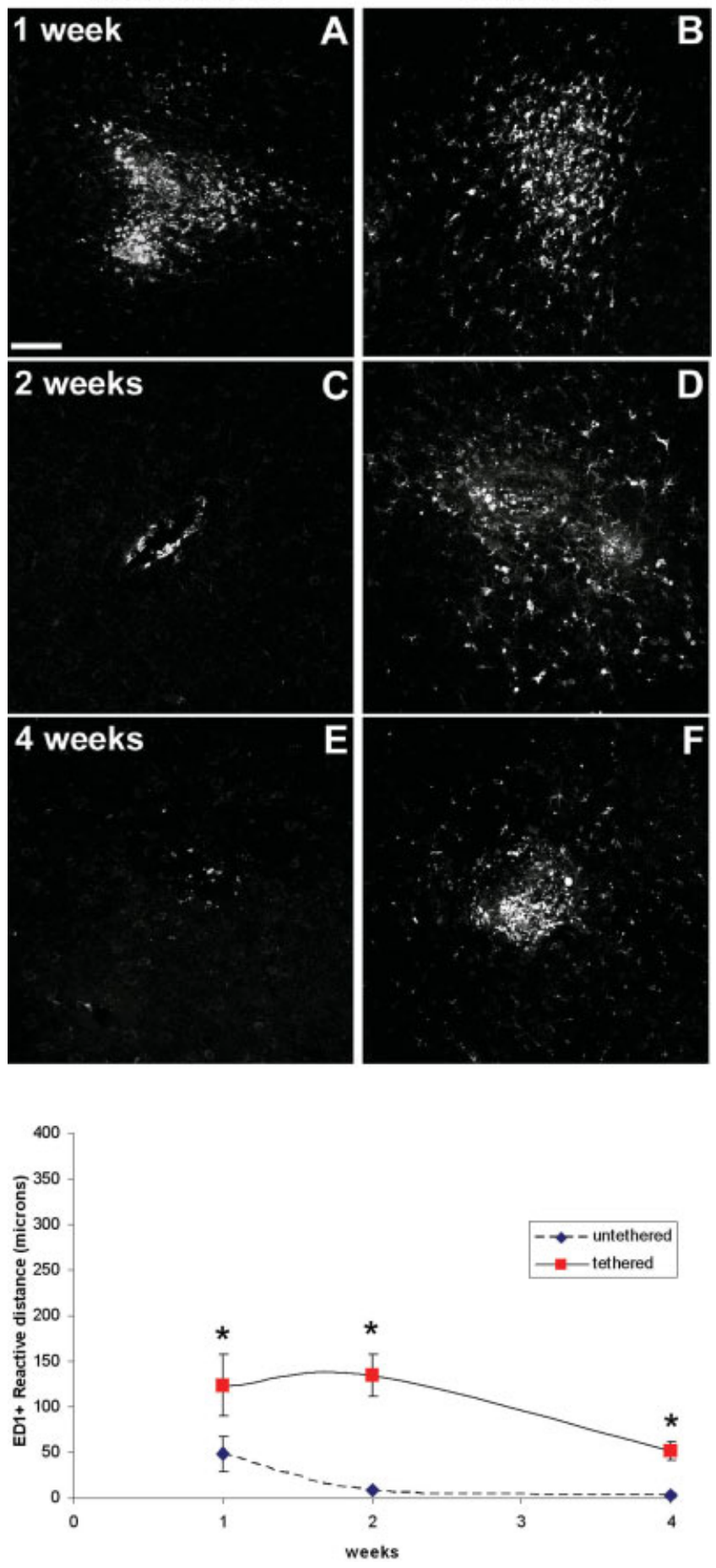

Figure 2. Comparison of the ED1+ spatial and temporal reactivity surrounding untethered and conventionally tethered silicon microelectrode arrays. Representative images of horizontal sections perpendicular to the implantation tract in the cortex of young adult rats receiving untethered $(\mathrm{A}, \mathrm{C}, \mathrm{E})$ or conventionally tethered $(B, D, F)$ microelectrode arrays at 1 week $(A, B), 2$ weeks $(\mathrm{C}, \mathrm{D})$, and 4 weeks $(\mathrm{E}, \mathrm{F})$ after implantation, illustrating an elevated $\mathrm{ED} 1$ immunoreactivity in brain tissue surrounding the implanted microelectrode arrays. The tissue sections shown were from the mid cerebral cortex. Scale bar $=100 \mu \mathrm{m}$. Lower panel summarizes the quantitative comparison of spatial and temporal distribution of elevated ED1. Asterisk indicates significant differences at a $p$ value $<0.05$. [Color figure can be viewed in the online issue, which is available at www.interscience.wiley.com.]


Figure 3. Comparison of elevated GFAP immunoreactivity as a function of distance surrounding the electrode over time. Representative images of horizontal tissue sections perpendicular to the electrode tract of untethered $(\mathrm{A}, \mathrm{C}, \mathrm{E})$ or conventionally tethered $(\mathrm{B}, \mathrm{D}, \mathrm{F})$ microelectrodes at 1 week $(A, B), 2$ weeks $(C, D)$, and 4 weeks $(E, F)$ after implantation, illustrating elevated reactivity around conventionally tethered microelectrodes relative to freely floating microelectrodes. Tissue sections were analyzed at the level of the cerebral cortex. Scale bar $=100 \mu \mathrm{m}$. Lower panel summarizes the quantitative comparison of spatial and temporal distribution of elevated GFAP. Asterisk indicates significant differences at a $p$ value $<0.05$. 
into a more compact zone as observed by others. ${ }^{16}$ As was observed for ED1, elevated GFAP reactivity persisted around the sites of both implant types over the course of the study.

\section{Retrieved microelectrodes are covered with macrophages}

Under light microscopy retrieved electrodes contained adherent material of cellular origin. In order to determine the identity of the attached cells, microelectrodes were immunostained with the same battery of antisera applied to tissue sections. Staining with DAPI revealed that all retrieved microelectrodes contained on both sides of the implant small cells with compact nuclei. The extent of electrode surface area covered by the cells varied between different electrodes, regardless of electrode type or the duration of the indwelling period. The adherent cells were almost entirely immunopositive for ED1 and MAC-1 (OX-42), indicating that they were activated microglia and hematogenous macrophages (Fig. 4). The adherent cell layers were similar in appearance to the cells observed immediately adjacent to the implant cavity in tissue sections. None of the electrodes contained neurofilament ${ }^{+}$material and only rarely was GFAP+ material detected. Where GFAP was observed, it was often in fragments that resembled astrocytic processes. No complete GFAP+ cells were observed. Furthermore, the GFAP-reactive material was never in direct contact with the electrode surface, rather it was attached to the surface of the macrophage/microglial cell layer.

\section{Reduced neuron density around microelectrodes}

The impact of electrode implantation on the surrounding neuronal population was assessed by immunostaining for neurofilament 160 , which in our hands stains rat brain cortical neuron dendrites, somas and axons, and NeuN, a nuclear antigen found only in neuronal cells. In the tethered cohort, tissue surrounding the electrode contained a reduced expression of neurofilament ${ }^{+}$immunostaining and $^{-}$ staining for NeuN (Fig. 5). In the untethered cohort, little loss of neurofilament 160 was observed especially at 4 weeks [Fig. 5(A)]. However, around tethered implants decreased neurofilament 160 was strikingly evident [Fig. 5(B)]. The extent of reduction varied with the dorsal/ventral aspect of the implant. In tethered implants, we observed reductions in neurofilament reactivity extending as far away as $250 \mu \mathrm{m}$, but on average the reduction was in a zone of $100 \mu \mathrm{m}$ around the implant site as previously described (Fig. 5, bottom panel). ${ }^{18}$

The distance between the electrode interface and the nearest $\mathrm{NeuN}+$ cell body appeared correlated to
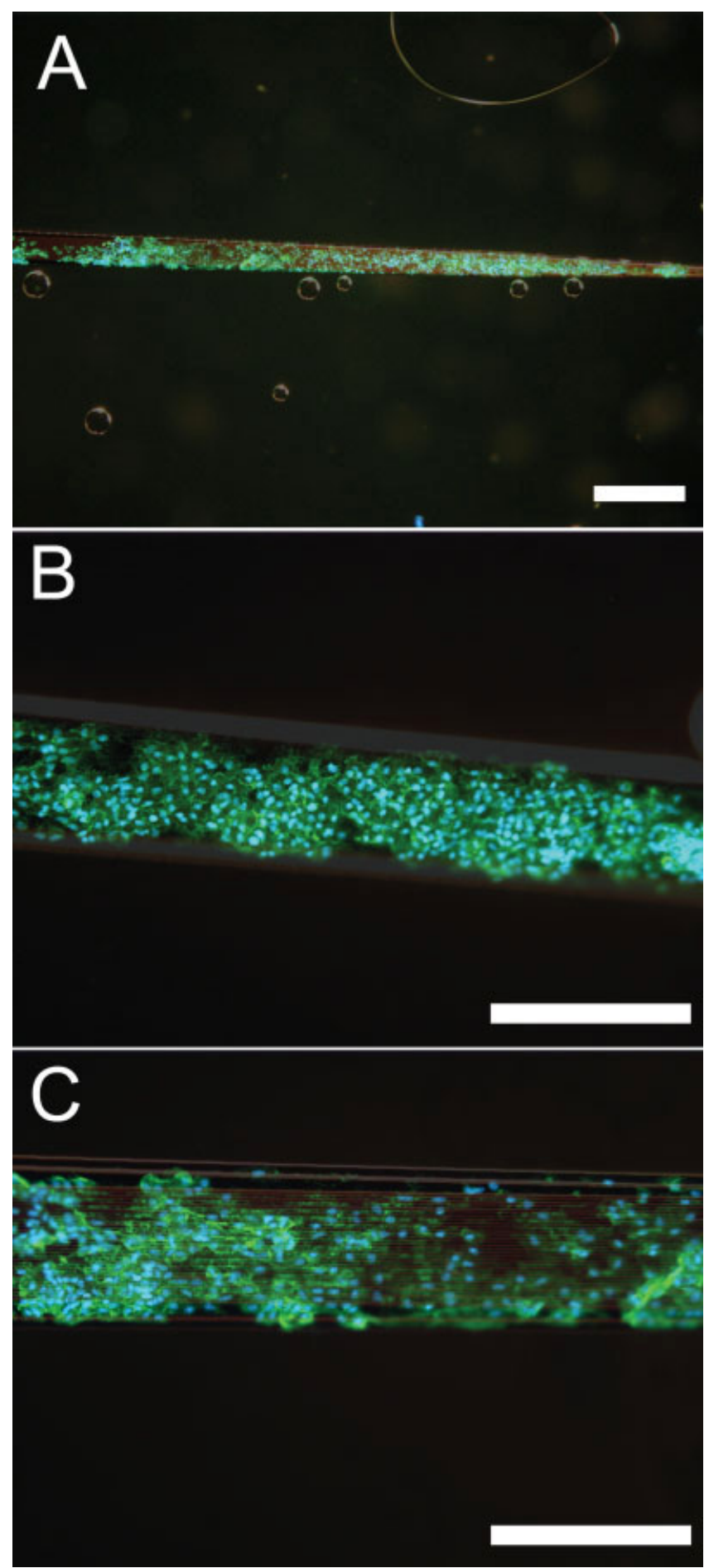

Figure 4. Cell coated retrieved planar silicon microelectrode arrays. (A-C) Representative images of microelectrode arrays staining positive with antibodies directed against ED1, an antigen expressed predominantly on lysosomal membranes of macrophages, but not found in normal brain tissue at $\times 4(\mathrm{~A}), \times 10(\mathrm{~B})$, and $\times 20(\mathrm{C})$. The nuclei of cells is shown stained blue with DAPI. Scale bar $=100 \mu \mathrm{m}$. [Color figure can be viewed in the online issue, which is available at www.interscience.wiley.com.] 

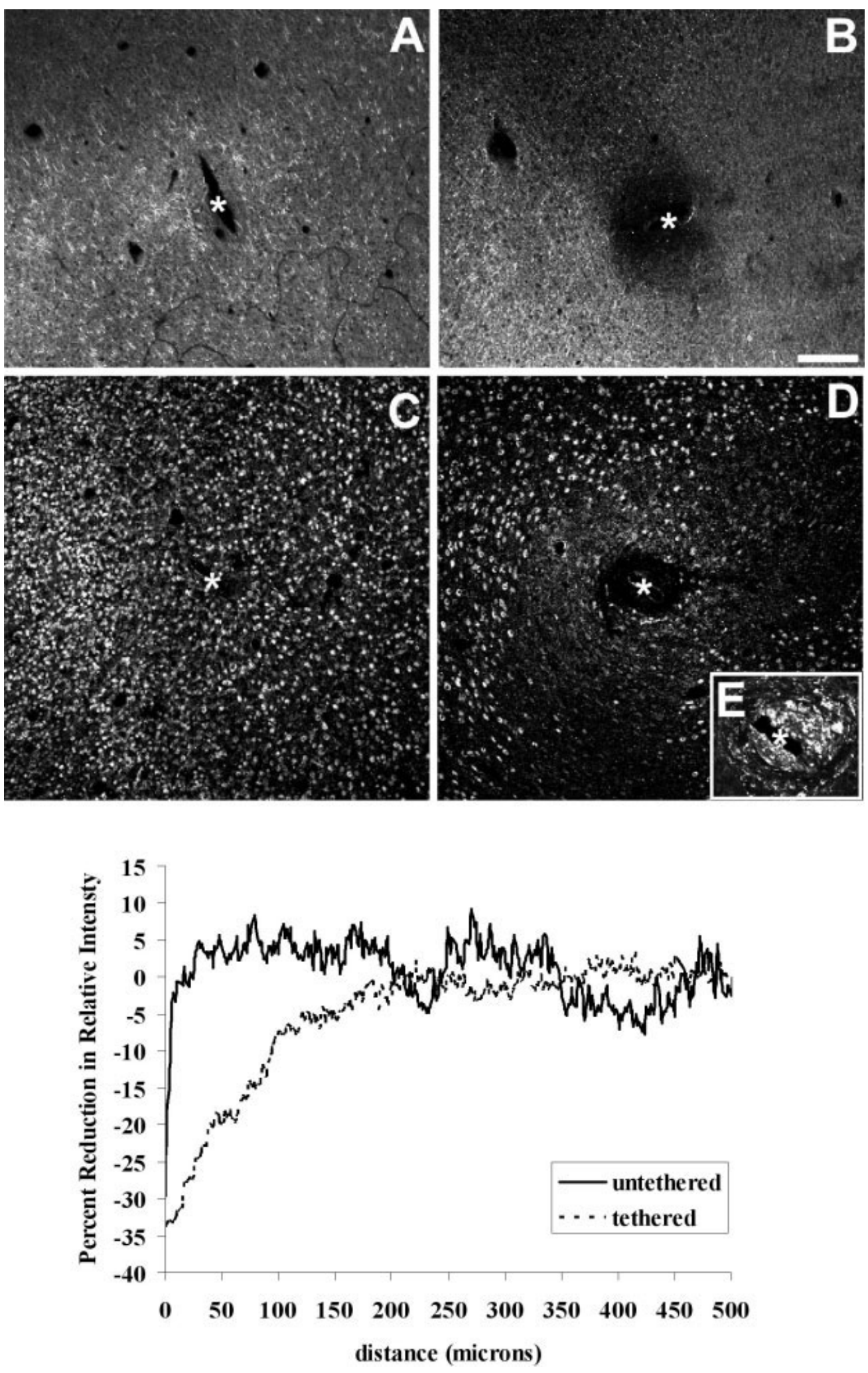

Figure 5. Representative horizontal images showing neurofilament and neuronal cell bodies distribution around implantation tracts at mid cortical level at 4 weeks after implantation. A comparison of neurofilament 160 immunostaining between untethered (A) and tethered microelectrodes (B). Immunostaining with the NeuN antibody demonstrates reduced neuronal bodies around tethered microelectrodes (D) compared with untethered controls (C). The inset in (E) shows the area around the implantation tract shown in (D) double-stained for ED1 showing the presence of macrophages. Reduction in neuronal cell body density correlated with a high degree of inflammation and was minimal in the untethered group. Scale bar $=100 \mu \mathrm{m}$, and asterisks $\left(^{*}\right)$ mark the location of the microelectrode tract. Lower panel provides a comparison of the average cohort neurofilament reactivity profile as a function of distance from the electrode after 4 weeks after showing minimal reduction in the untethered group and a greater reduction in the tethered cohort as previously reported. ${ }^{18}$ 
the severity of the macrophage reaction. Near untethered implants where the spatial extent of ED1 immunoreactivity was low, NeuN+ neuronal bodies were found adjacent to the microelectrode brain tissue interface [Fig. 5(C)]. In contrast, NeuN+ neuronal cell bodies were much farther away from tethered microelectrodes that exhibited a larger ED1 response [Fig. 5(D,E)].

\section{DISCUSSION}

The current study demonstrates that the method of anchoring silicon micromachined electrode arrays to the skull significantly influences the brain tissue reaction, including greater macrophage/microglial activation, increased astrogliosis, and a decrease in immunoreactivity against neurofilament and NeuN at the device brain tissue interface. Increased reactivity around tethered microelectrodes may result from a number of variables including the differing surgical procedure, tethering forces, and the provision of a pathway for meningeal cell migration. Similarities in the spatial and temporal characteristics of the response were in agreement with previous reports. ${ }^{15,16}$ Regardless of microelectrode anchoring technique, a response involving macrophages persisted at the electrode interface over the course of the study and retrieved microelectrodes contained to varying degrees adherent macrophages on the device surface.

An interesting finding was the reduction in neuronal density as well as the decrease in neuronal bodies at the electrode brain tissue interface, which was greatest in the tissue immediately surrounding highly reactive implant sites. In some tethered specimens, this reduction extended beyond the "kill zone" previously reported using another electrode implantation paradigm. ${ }^{12}$ In the current study, reduced nerve fiber and soma density coincided with relatively higher levels of ED1 immunoreactivity, an indicator of inflammation. The reduction in neuronal density was variable between animals within a group and variable along the dorsal ventral axis of a given implant, perhaps related to differences in local neuroanatomy, micromotion, or tethering forces.

It is possible especially at the early time points that we were observing degenerating axons that were mechanically severed during implantation. Indeed, the appearance of swollen neuronal fibers around many electrode sites resembled the retraction bulbs characteristic of severed axons described as early as 1959 by Ramon and Cajal. ${ }^{22}$ Furthermore, our observations are also consistent with the fact that activated microglia accumulate at sites of nerve fiber degeneration after axotomy. ${ }^{23}$ However, the larger reduction in neuronal density around tethered microelectrodes is unlikely to be due to implant- induced axotomy alone, as no such observations were made around untethered microelectrodes and stab wound controls. ${ }^{18}$ The extent to which the presence of macrophage and elevated microglial activation contributed to neuronal damage is unclear. Nevertheless, the functional implications of these observations on recording performance may be very important. It appears that in certain instances, neuronal death and degeneration take place over distances that span the effective recording range of this type of microelectrode. ${ }^{6}$ The variability of our observations may explain, in part, the inconsistent nature of long-term recordings using different hardware configurations and implantation schemes.

Using a different CNS implantation paradigm, Kim et al. ${ }^{24}$ described increased microglial and astrocyte reactivity around larger tubular implants. While the size of the implants was substantially larger than the microelectrodes employed in the current study, a similar pattern of reactivity was observed. Several important differences between the Kim et al. study and the present study deserve mention. For example, the silicon microelectrode arrays used in the present study have an elastic modulus that is several orders of magnitude stiffer than the thermoplastic employed in the Kim et al. study. The surface architecture was also significantly different. In the present study, the silicon surface was relatively flat on the nanometer level while the hollow fiber used in the Kim et al. study possessed macroscopic holes and presented orders of magnitude larger surface area. Additionally, the footprint or overall size of the device in the Kim et al. study was an order of magnitude larger than the implants studied here. Even with all of these differences, the general appearance of the foreign body response was similar. In both cases the elevation of ED1 was restricted to the biotic-abiotic interface and the elevation of GFAP extended only several hundred micrometers, suggesting that the extent of tissue reactivity does not necessarily scale with the size of the device but rather appears to be a product of the interface. Clearly this requires further study under better controlled conditions. Taken together, these observations suggest a reproducible influence of anchorage on the tissue response adjacent to devices implanted in the CNS and suggests that the reaction is independent of the specific implant material or its dimensions.

While the precise mechanisms responsible for the increased tissue reactivity to tethered implants are unknown, several explanations appear reasonable including: (1) shearing and/or compression of adjacent tissue due to the stiffness mismatch of silicon implants and brain tissue caused by relative motion between the skull and the brain itself, and (2) the potential migration and colonization of the electrode interface by fibroblasts from the overlying 
meninges. ${ }^{24}$ We have frequently observed histopathological evidence suggesting movement of tethered electrodes within cortical tissue, characterized by narrow and elongated cavities that are much larger than the corresponding cross section of the microelectrode. Such movement would clearly result in a wider zone of inflammation and tissue reactivity. If such mechanisms are at play, it follows that it may be possible to improve microelectrode biocompatibility by addressing the issue of implant movement and fibroblast infiltration through modifications of the electrode surface, changes in the tethering approach, or to devise microelectrode implantation strategies which avoid cranial tethering altogether. It is also possible that the irregularly shaped reactions are caused by asymmetric degeneration of dendrites or axonal projections that occur along the implantation tract. Clearly more detailed studies are required to shed light on the precise mechanisms responsible for these observations.

In general, the major difference in tissue reactivity between tethered microelectrodes and untethered microelectrodes was in magnitude rather than the composition and arrangement of the tissue adjacent to the implant. Around all microelectrodes we observed a stratified cellular response composed of an activated zone of microglia attached to and residing most proximal to the microelectrode shaft and a layer of hypertrophied intensely GFAP+ reactive astrocytes surrounding the macrophages, and finally, a somewhat normal parenchyma that included a normal arrangement of neuronal bodies. Within the zone of reactivity we observed a reduction of neuronal processes and nerve cell bodies.

There is no evidence from our study, and from later time points in other studies examining the brain tissue reaction to implanted silicon microelectrodes, ${ }^{16,19}$ that the macrophage phenotype (ED1+) disappears from the electrode-brain tissue interface. The persistence of this phenotype at the interface contrasts with the relatively rapid disappearance of activated microglia and macrophages near cortical stab wounds as previously reported ${ }^{25}$ and as observed following microelectrode stab wounds made by electrodes. ${ }^{18}$ Aside from supporting the widely acknowledged phenomenon that nondegrading implants elicit a chronic foreign body reaction, the observation of a persistently activated macrophage response at electrode-brain tissue interface raises the question of whether such a response can have longterm functional implications for recording performance of neuroprosthetic devices.

Growing evidence suggests that persistently activated microglia, such as those found around insoluble plaques in Alzheimer's disease, ${ }^{26}$ may be a source of local neurotoxicity. In the CNS, like in the periphery, it is has been observed that the inability of macrophages to clear insoluble material results in a phenomenon described as "frustrated phagocytosis" which is characterized by increased secretion of inflammatory products that may directly or indirectly cause neuronal death or substantially alter synaptic activity. ${ }^{27}$ Often these secreted products also perpetuate microglial activation through autocrine stimulation and by further promoting adjacent cell death. Microglial-induced neuronal death can be mediated by a myriad of secreted compounds. The most notable includes various complement components that can cause neuronal lysis, ${ }^{28-30}$ such as cytokines TNF $\alpha$ and IL-1 $\beta$, which have direct and indirect mechanisms of action, ${ }^{27}$ prostaglandins that indirectly contribute to excitotoxic neuronal death, ${ }^{31}$ as well as superoxide anion ${ }^{32}$ and elevated nitric oxide. ${ }^{33,34} \mathrm{TNF} \alpha$, perhaps the archetypal pro-inflammatory cytokine, is secreted at high levels by activated microglia. ${ }^{35,36}$ It has been shown that TNF $\alpha$, in the presence of activated microglia, mediates excitotoxic neuronal death by causing extensive release of glutamate from adjacent astrocyte, ${ }^{37}$ and has recently been shown to be released by the cells attached to retrieved silicon microelectrode arrays. ${ }^{18}$ Even without causing neuronal death, elevated but sublethal doses of this and other microglial-derived agents can alter neuronal activity ${ }^{27,37}$ and thereby reduce the flow of electrical activity within the cortical column.

In light of or our own observations, it is tempting, therefore, to hypothesize that such events may also take place around all chronically indwelling implants regardless of material composition. Whether in fact there exists a consistent pattern of neuronal death surrounding chronically implanted devices remains uncertain and will require further investigation. It is also possible, by the very probability that one electrode tract may cause more or less tissue trauma than another (for example, by variation in the degree of vascular trauma or variations in local neuroanatomy), that the degree of neuronal damage is naturally a highly variable outcome and may in part explain the variable nature of achieving successful chronic single unit recording.

\section{CONCLUSIONS}

The current study demonstrates that the manner that a recording device in anchored to the skull alone can increase the brain reaction to implanted microelectrode arrays. The increased reactivity surrounding conventionally tethered microelectrodes may be due, in part, to variables other than the biomaterials alone and includes the presence of acrylic resin and bone screws used to fix the electrode, as well as the anchoring mechanisms and tethering forces involved. Our study also suggests that the persistence of macrophages surrounding chronically im- 
planted neuroprosthetic devices may have deleterious effects on adjacent nerve cell bodies and their processes, and therefore represents another potentially important mechanism that may be responsible for decreased performance and reliability of this emerging class of biomedical technology and represent a therapeutic target for enhancing their biocompatibility.

The authors gratefully acknowledge Ms. Lindsay Alder, Ms. Mindy Naegle, and Dr. Elena Budko for their technical assistance.

\section{References}

1. Nicolelis MA, Ribeiro S. Multielectrode recordings: The next steps. Curr Opin Neurobiol 2002;12:602-606.

2. Edgerton BJ, House WF, Hitselberger W. Hearing by cochlear nucleus stimulation in humans. Ann Otol Rhinol Laryngol Suppl 1982;91(2, Part 3):117-124.

3. Benabid AL, Pollak P, Gervason C, Hoffmann D, Gao DM, Hommel M, Perret JE, de Rougemont J. Long-term suppression of tremor by chronic stimulation of the ventral intermediate thalamic nucleus. Lancet 1991;337:403-406.

4. Benabid AL, Pollak P, Gao D, Hoffmann D, Limousin P, Gay E, Payen I, Benazzouz A. Chronic electrical stimulation of the ventralis intermedius nucleus of the thalamus as a treatment of movement disorders. J Neurosurg 1996;84:203-214.

5. Kruger J. Simultaneous individual recordings from many cerebral neurons: Techniques and results. Rev Physiol Biochem Pharmacol 1983;98:177-233.

6. Drake KL, Wise KD, Farraye J, Anderson DJ, BeMent SL. Performance of planar multisite microprobes in recording extracellular single-unit intracortical activity. IEEE Trans Biomed Eng 1988;35:719-732.

7. Branner A, Stein RB, Normann RA. Selective stimulation of cat sciatic nerve using an array of varying-length microelectrodes. J Neurophysiol 2001;85:1585-1594.

8. Liu X, McCreery DB, Carter RR, Bullara LA, Yuen TG, Agnew WF. Stability of the interface between neural tissue and chronically implanted intracortical microelectrodes. IEEE Trans Rehabil Eng 1999;7:315-326.

9. Schultz RL, Willey TJ. The ultrastructure of the sheath around chronically implanted electrodes in brain. J Neurocytol 1976;5:621-642.

10. Schmidt EM, Bak MJ, McIntosh JS. Long-term chronic recording from cortical neurons. Exp Neurol 1976;52:496-506.

11. Agnew WF, Yuen TG, McCreery DB, Bullara LA. Histopathologic evaluation of prolonged intracortical electrical stimulation. Exp Neurol 1986;92:162-185.

12. Edell DJ, Toi VV, McNeil VM, Clark LD. Factors influencing the biocompatibility of insertable silicon microshafts in cerebral cortex. IEEE Trans Biomed Eng 1992;39:635-643.

13. Schmidt S, Horch K, Normann R. Biocompatibility of siliconbased electrode arrays implanted in feline cortical tissue. J Biomed Mater Res 1993;27:1393-1399.

14. McCreery DB, Yuen TG, Agnew WF, Bullara LA. A characterization of the effects on neuronal excitability due to prolonged microstimulation with chronically implanted microelectrodes. IEEE Trans Biomed Eng 1997;44:931-939.

15. Turner JN, Shain W, Szarowski DH, Andersen M, Martins S, Isaacson $\mathrm{M}$, Craighead $\mathrm{H}$. Cerebral astrocyte response to micromachined silicon implants. Exp Neurol 1999;156:33-49.

16. Szarowski DH, Andersen MD, Retterer S, Spence AJ, Isaacson M, Craighead HG, Turner JN, Shain W. Brain responses to micro-machined silicon devices. Brain Res 2003;983:23-35.
17. Roitbak T, Sykova E. Diffusion barriers evoked in the rat cortex by reactive astrogliosis. Glia 1999;28:40-48.

18. Biran R, Martin DC, Tresco PA. Neuronal cell loss accompanies the brain tissue response to chronically implanted silicon microelectrode arrays. Exp Neurol 2005;195:115-126.

19. Schwartz AB. Cortical neural prosthetics. Annu Rev Neurosci 2004;27:487-507.

20. Bauer J, Sminia T, Wouterlood FG, Dijkstra CD. Phagocytic activity of macrophages and microglial cells during the course of acute and chronic relapsing experimental autoimmune encephalomyelitis. J Neurosci Res 1994;38:365-375.

21. Mathewson AJ, Berry M. Observations on the astrocyte response to a cerebral stab wound in adult rats. Brain Res 1985; 327:61-69.

22. Ramon Y, Cajal S. Degeneration and Regeneration of the Nervous System. New York: Hafner; 1959. 761 p.

23. Perry VH, Brown MC, Gordon S. The macrophage response to central and peripheral nerve injury. A possible role for macrophages in regeneration. J Exp Med 1987;165:1218-1223.

24. Kim YT, Hitchcock RW, Bridge MJ, Tresco PA. Chronic response of adult rat brain tissue to implants anchored to the skull. Biomaterials 2004;25:2229-2237.

25. Berry M, Maxwell WL, Logan A, Mathewson A, McConnell P, Ashhurst DE, Thomas GH. Deposition of scar tissue in the central nervous system. Acta Neurochir Suppl 1983;32:31-53.

26. Luber-Narod J, Rogers J. Immune system associated antigens expressed by cells of the human central nervous system. Neurosci Lett 1988;94:17-22.

27. Hanisch UK. Microglia as a source and target of cytokines. Glia 2002;40:140-155.

28. Gasque P, Dean YD, McGreal EP, VanBeek J, Morgan BP. Complement components of the innate immune system in health and disease in the CNS. Immunopharmacology 2000; 49:171-186.

29. McGeer PL, Akiyama H, Itagaki S, McGeer EG. Activation of the classical complement pathway in brain tissue of Alzheimer patients. Neurosci Lett 1989;107:341-346.

30. Singhrao SK, Neal JW, Rushmere NK, Morgan BP, Gasque P. Spontaneous classical pathway activation and deficiency of membrane regulators render human neurons susceptible to complement lysis. Am J Pathol 2000;157:905-918.

31. Rothstein JD, Jin L, Dykes-Hoberg M, Kuncl RW. Chronic inhibition of glutamate uptake produces a model of slow neurotoxicity. Proc Natl Acad Sci USA 1993;90:6591-6595.

32. Colton CA, Chernyshev ON, Gilbert DL, Vitek MP. Microglial contribution to oxidative stress in Alzheimer's disease. Ann NY Acad Sci 2000;899:292-307.

33. Banati RB, Graeber MB. Surveillance, intervention and cytotoxicity: Is there a protective role of microglia? Dev Neurosci 1994;16:114-127.

34. Liu B, Gao HM, Wang JY, Jeohn GH, Cooper CL, Hong JS. Role of nitric oxide in inflammation-mediated neurodegeneration. Ann NY Acad Sci 2002;962:318-331.

35. Rostworowski M, Balasingam V, Chabot S, Owens T, Yong VW. Astrogliosis in the neonatal and adult murine brain post-trauma: Elevation of inflammatory cytokines and the lack of requirement for endogenous interferon-g. J Neurosci 1997;17:3664-3674.

36. Bruccoleri A, Brown H, Harry GJ. Cellular localization and temporal elevation of tumor necrosis factor- $\alpha$, interleukin- $1 \alpha$, and transforming growth factor- $\beta 1 \mathrm{mRNA}$ in hippocampal injury response induced by trimethyltin. J Neurochem 1998; 71:1577-1587.

37. Bezzi P, Domercq M, Brambilla L, Galli R, Schols D, De Clercq E, Vescovi A, Bagetta G, Kollias G, Meldolesi J, Volterra A. CXCR4-activated astrocyte glutamate release via TNF $\alpha$ : Amplification by microglia triggers neurotoxicity. Nat Neurosci 2001;4:702-710. 